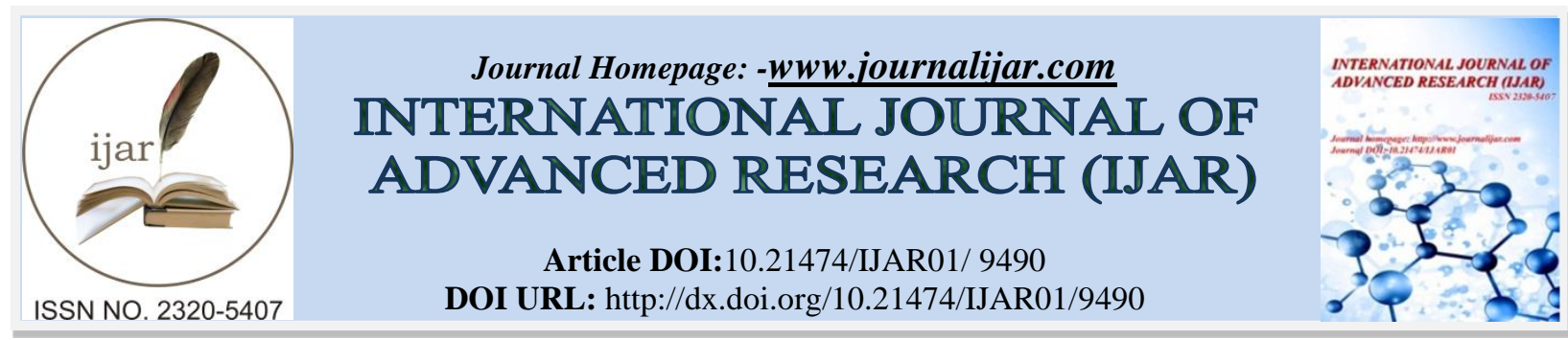

RESEARCH ARTICLE

\title{
EFFICACY OF KINESIO-TAPING OVER LEVATOR SCAPULAE TRIGGER POINT ON PAIN, PRESSURE PAIN THRESHOLD AND FUNCTION IN CHRONIC MECHANICAL NECK PAIN: A RANDOMIZED CONTROLLED TRIAL.
}

Swarn Lata, Pravin Kumar and Bibhuti Sarkar ${ }^{3}$.

Professional Trainee (PT), Assistant professor (PT), Demonstrator (PT); Department of Physiotherapy, NationalInstitute for Locomotor Disabilities (Divyangjan), B.T.Road, Bonhoogly, Kolkata-700090, West Bengal, India.

\section{Manuscript Info}

(n.........................

Manuscript History

Received: 05 June 2019

Final Accepted: 07 July 2019

Published: August 2019

Key words:-

Chronic Mechanical Neck Pain, Levator scapulae trigger point, Kinesio Taping, standardized exercise.

\section{Abstract}

Introduction: Chronic Mechanical Neck Pain (CMNP) is one of the most common health problems. Patient with neck pain were found to have the highest prevalence rate of myofascial trigger points.

Subjects And Methods: Forty-five (45) subjects with CMNP were randomly assigned into three groups. Subject in Group-A ( $\mathrm{n}=15)$ with mean age of $29.47 \pm 5.85$ received Kinesio Taping (KT), standardized exercise (SE) protocol \& home exercise program (HEP), Group- B $(\mathrm{n}=15)$ with mean age of $31.53 \pm 7.80$ received sham Kinesio taping (SKT), SE protocol \& HEP \& Group-C control group $(\mathrm{n}=15)$ with mean age of $32.00 \pm 8.37$ received SE protocol \& HEP received 5 sessions 2 session per week \& HEP was advised for every day for 2 weeks. Pain intensity was measured by VAS, PPT was measured by Algometer \& Function was measured by NDI were obtained at baseline and after completion of 5 session.

Results: All the three groups were homogeneous at baseline as there was non-significant $(p>0.05)$ difference among groups related to all parameters. All the groups showed statistically significant improvement after 5 session of intervention in respect to VAS, PPT \& NDI in CMNP. But when compared among the groups group A \& group B showed significant $(\mathrm{p}<0.05)$ improvement in PPT when compared to Group $\mathrm{C}$ where as other parameters showed non-significant $(\mathrm{p}>0.05)$ difference among the groups after 5 session of treatment.

Conclusion: The results of this study suggested that KT with SE protocol and SKT with SE protocol has a significant effect in improving pressure pain threshold whereas there was non-significant difference found among group on VAS \& NDI in subjects with levator scapulae trigger point in CMNP.

Copy Right, IJAR, 2019,. All rights reserved.

\section{Corresponding Author:-Swarn Lata.}

Address:- Professional Trainee (PT), Assistant professor (PT), Demonstrator (PT);Department of Physiotherapy, NationalInstitute for Locomotor Disabilities (Divyangjan),B.T.Road, Bonhoogly, Kolkata-700090, West Bengal, India. 


\section{Introduction:-}

Cervical spinal pain defined by the International Association for the Study of Pain as "pain perceived anywhere in the posterior region of the cervical spine, from the superior nuchal line to the first thoracic spinous process" and pain can be located in the anatomical region of the neck with or without radiation to the head, trunk, and upper limbs". Pain is classified as chronic when it has a duration of 12 weeks or more. ${ }^{1}$

Neck pain is common throughout the world which is affecting individuals and their families, communities, healthcare systems, and businesses. Neck pain is a common disorder which it is estimated to affect around 50-67\% of the population at some point of their lives, with the subsequent associated costs. ${ }^{2}$ Prevalence is generally higher in women, with a higher incidence noted in office and computer workers, higher in high-income countries compared with low- and middle-income countries and higher in urban areas compared with rural areas. ${ }^{3}$

The origin of neck pain is thought to be multifactorial. Excessive physical strain may cause microtrauma in connective tissues, and psychosocial stress may lead to increased muscular tension. ${ }^{4}$ Rajwant et al (2015) in their study showed a significant increased level of EMG activity in neck muscle during performance of static activities in nontraumatic chronic neck pain. ${ }^{5}$ Lowered pressure pain thresholds, compared to healthy controls, have been observed in patients with chronic non-specific neck pain locally at the cervical spine. ${ }^{6}$

Within spinal pain, patient with neck pain were found to have the highest prevalence rate of Myofascial Trigger Points (MTrPs). The trapezius descenders, levator scapulae, and sub-occipital muscle were most prevalent location for active MTrPs in patient with neck pain. ${ }^{7}$ Often Levator scapulae muscle become tense and painful leading to reduce motion in the area. MTrPs are characterized by local tenderness with or without referred pain and also by muscle dysfunction (weakness, fatigue, stiffness, and poor blood flow), restricted range of motion (ROM), change of motor pattern, poor posture, and limited physical, professional, and social activity. ${ }^{8}$ Referred pain from the levator scapulae concentrates in the angle of the neck with a spill over zone along the vertebral border of the scapula and to the shoulder posteriorly. This "stiff neck" muscle, when involved, consistently limits neck rotation due to pain on movement. ${ }^{9}$

There are many ways to treat myofascial pain. The treatment can contain noninvasive methods like educational programs, behavioral cognitive therapy, medication, and physical therapy or physiotherapy (spray and stretch, general exercises, myofascial release, massage, Jacobson's muscle relaxation, autogenic training, manual therapy, neuromuscular techniques, electrotherapy-ultrasound, interferential therapy, transcutaneous electrical nerve stimulation, pulsed shortwave therapy, and laser therapy). ${ }^{10}$ More and more publications suggest that kinesio taping (KT) may be a new treatment option and indicate the possibility of the use of KT in patients with musculoskeletal problems, including MTrPs.

KT is a therapeutic taping technique developed by Dr. Kenzo Kase (Japan,1979). This technique uses an elastic tape that is thin and more elastic than conventional bandages. The tape can be stretched to $140 \%$ of its original length and applied to the skin. In addition, in the literature it can be found that KT can increase the ROM, reduce swelling, inflammation, and bruising, enhance blood circulation, enhance strength and muscle tone, or be used in muscle spasms and cramping prevention and to speed recovery of overused muscles. Most of the research is related to the use of KT in relieving pain, specifically reducing pain and disability. ${ }^{8}$

In a systemic review done by Mansour Alotaibi et al (2018) suggested that KT with other therapeutic protocols is effective in increasing ROM \& function in case of myofascial pain syndrome in upper trapezius. ${ }^{11}$ EL-ABD AM et al (2017) founded that KT has been found to be more effective than postural exercises to reduce neck pain. However, both modalities have similar effects to reduce axio-scapular muscles activation. ${ }^{12}$

In this randomised controlled trial, we aimed to compare the efficacy of KT, sham Kinesio taping and standardized exercise on Levator scapulae trigger point, pressure pain threshold and neck disability index in chronic mechanical neck pain.

\section{Material and Methods :-}

The study was conducted at National Institute for Locomotor Disabilities, Kolkata and data were collected between March 2018 to February 2019. This study was approved by the Institutional Ethics Committee of National Institute 
for Locomotor Disabilities, Kolkata. Subjects with complain of both genders with presence of at least one active trigger point located in levator scapula muscle (symptoms of at least 3 months duration), age between 18 - 45 years and visual analogue scale score between 3-8 were included in this study. Subject with history of neck and shoulder surgery, Recent trigger point injection or participating in a physical treatment program within the last three months, Radiculopathy, Cervical disc lesion, Diagnosis of fibromyalgia syndrome, Myelopathy, Neurological disorder affecting cervico-thoracic region and inflammatory diseases, Diabetic Neuropathy, Cardiovascular disease, Local skin disorder, Contraindications to the use of Kinesio Taping (allergy or intolerance) \& Patient on psychiatric medication and uncooperative were excluded in this study. All subjects were explained in details about the study and a written informed consent in their preferred language were obtained from each subject.

Total 48 subjects were selected for this study who met inclusion/exclusion criteria and were randomly allocated into three different groups through Convenient sampling method. Subjects were blinded to the study and group allocation. Group-A $(\mathrm{n}=16)$ Experimental group received (kinesio taping along with standardized Exercise protocol $\&$ home exercise program (HEP)) for 5 treatment session twice weekly. Group-B $(n=16)$ received (sham kinesio taping along with standardized Exercise protocol \& home exercise program) whereas Group-C $(n=16)$ control group received (standardized exercise protocol \& home exercise program). All group completed 5 session of treatment twice weekly. Total three subjects were drop out one from each group due to personal reason during study.

Outcome parameters were pain intensity measured by VAS, Pressure Pain Threshold (PPT) measured by Algometer and function by Neck Disability index (NDI). The data for all outcome measures were collected pre-intervention and after completion 5 session.

VAS is a straight horizontal line of fixed length usually $10 \mathrm{~cm}$. The VAS score is determined by measuring in millimetres from the left-hand end of the line to the point that the patient marks. Here 0 denote no pain and 10 denote worst pain.

PPT is determined using a hand-held pressure algometer with a $1 \mathrm{~cm}^{2}$ probes area with an increasing of the pressure rate of 20Kpa/s. The pressure algometer consists of a "pistol" handle \& rod with a pressure-sensitive gauge strain at the tip. Pressure algometer was performed on the same day under quiet $\&$ non -stressful condition. The tip of the algometer is positioned on this specific point. By pushing the algometer, the force applied to that area gradually increased. The participants were not allowed to see the algometer in any moment $\&$ as soon as the volunteer experienced a painful sensation, they said "stop", the algometer is immediately released \& the force (Kpa) is read from the display.

The NDI contains seven items related to activities of daily living, two items related to pain, and one item related to concentration (ability to read). Each item is scaled from 0 to 5 , and the total score is expressed as a percentage, with higher scores representing greater levels of disability. The maximum total score is 50 points, and the minimum of 0 implies no disability. The test can be interpreted as a raw score, with a maximum score of 50, or as a percentage.

In Group A, manual stretching of levator scapulae \& manual neck isometric exercise were given to the subject prior to the application of kinesio tape. After standardized exercise, kinesio tape were applied to levator scapulae muscle. The shoulder was depressed and neck was in lateral flexion and rotation position to the opposite side. The kinesio tape was placed on the same muscle using four "I" strips' application in star shape to create more space directly above an area of pain (space correction). Each strip was stretched to $50 \%$ of available tension.

In Group B, after standardized exercise sham- kinesio tape was applied with four "I" strips' application in star shape of the same material on levator scapulae of the muscle without a tension with the neck in neutral position.

In Group C, subjects were receiving only standardized exercise. And home exercise program was taught in all the groups.

\section{Statistical Analysis :-}

The statistical analysis of the gathered data was analysed by using SPSS software (version 23). Baseline characteristics of the non-parametric data between the three groups were measure by using Kruskal-Wallis test and parametric data between the three groups were measure by using ANOVA. Paired sample $t$ test was used to analyse within group variables for VAS, PPT and NDI and ANOVA was used to compare among groups. Baseline \& post- 
treatment measurements in each group were performed by ANOVA with post hoc Bonferroni test. The tests were applied at $95 \%$ confidence interval on $\alpha$ value set at 0.05 . The results were taken to be significant if $\mathrm{P}$-value $<0.05$.

\section{Results:-}

Total 45 subjects were included in this study. All the three groups were homogenous at the time of start of study as there was not significant difference ( $\mathrm{p}>0.05$ ) among groups related to age, gender, VAS, PPT \& NDI. (Table- 1) Intragroup comparison showed that there was statistically significant improvement after 5 session of intervention in all the groups in respect to VAS, PPT \& NDI in CMNP. (Table-2)

But when compared among the groups group A \& group B showed significant $(\mathrm{p}<0.05)$ improvement in PPT when compared to Group $\mathrm{C}$ where as other parameters showed non-significant $(\mathrm{p}>0.05)$ difference among the groups after 5 session of treatment. (Table- 3 )

\section{Discussion:-}

The purpose of this study was to investigate the efficacy of Kinesio Taping with standardized exercise, sham Kinesio taping with standardized exercise and standardized exercise alone over Levator scapulae trigger point on pain, pressure pain threshold and function in chronic mechanical neck pain. The result of this study showed statistically significant improvement after 5 session of intervention in respect to pain intensity, pressure pain threshold and function in Levator scapulae trigger point in Chronic Mechanical Neck Pain in all the three groups but when we compared among the three groups, there was non-significant different found in pain (VAS) \& NDI. However, there was significant difference found in PPT.

Group A received Kinesio Taping with standardized exercise and reason of reduction of pain perception \& neck function in this group may be due to KT \& exercise. Application of KT which enhanced proprioception, cutaneous mechanoreceptors, improved blood and lymphatic circulation, reduced pain severity, realignment of joints, assist the postural alignment and relax the overused muscles. ${ }^{13}$ The reason behind reduction of pressure pain threshold in Group A may be due to application of kinesio tape over levator scapulae trigger point. In present study, KT was attached in stretched position of muscle fiber. ${ }^{14}$ This position corrects sarcomere length in TrP and decreases pain and spasm. Halski (2015) stated that KT increases blood and lymphatic fluid circulation under the taped area in a consequence of a lifting effect, which creates a wider space between the skin and the muscle, what may affect muscle functions and result in pain and ROM improvement. ${ }^{8}$ Saime Ay (2017) showed that Kinesio Taping leads to improvements on pain, pressure pain threshold and cervical range of motion, but not disability in short time in cervical myofascial pain syndrome. ${ }^{13}$

Whereas Group B received sham Kinesio Taping with standardized exercise and sham taping was similar to that of real taping except that sham taping was applied as tension free. Reduced pain perception, pressure pain threshold \& neck function after treatment in the sham KT group would be due to placebo effect which is considered to be the results of possible psychological effects. The placebo application was improper, it was applied to the same muscle so it probably provided proper sensory feedback during cervical spine movements, which decreased mechanical irritation of the soft tissues. This sensory feedback may improve patient awareness and thus increase their adherence to the ergonomics principles as suggested by Ozturk et al (2016). ${ }^{15}$

However, in this study when it was compared among all the groups, Group A \& Group B (KT group and Sham KT group) was significantly superior to the Group $\mathrm{C}$ who received manual stretching and strengthening exercise of neck only in terms of pressure pain threshold in Levator Scapulae trigger point in chronic mechanical neck pain after 5 session of intervention. El-Abd (2017) in their study showed that Patients with mechanical neck pain performing postural correction exercises or receiving an application of KT exhibited similar reduction in axioscapular muscle activities. However, reduction of pain was greater in patients received KT. So KT may be an alternative treatment option in the treatment of Mechanical Neck Dysfunction. ${ }^{16}$

Subjects in Group C received standardized exercise and the possible reason behind that would be the standardized exercise in which patients were receiving stretching and strengthening exercise. Thomas et al. (2004) suggested that as the disability score aims to assess different aspects of the clinical symptoms of neck pain (which consist of pain 
intensity, daily activities, work, and social activities), the improvement in disability score might be due to the combined effects of reduction in neck pain, improvement in neck muscle strength and to certain extent improvement in activities of daily living could be a result of the exercise program. ${ }^{17}$

When it was compared among all the three group there was no statistically significant difference found among the groups in neck disability in this study. There are several studies which have shown that kinesio tape and sham kinesio tape was not superior to the strengthening and stretching exercises. Saime et al (2017) in their study showed that KT is a non-invasive, painless method that has fewer side effects, is well tolerated and has been used in MPS. This study shows that KT leads to improvements on pain, PPT, and ROM, but not in disability in short period. Therefore, KT can be used as an alternative therapy in the treatment of patients with MPS. ${ }^{13}$ Similar result was found in a systematic review done by Parreira et al who concluded that comparison of KT with sham taping either provided no significant benefit or its effect was too small to be clinically worthwhile. ${ }^{18}$

\section{Conclusion:-}

The results of this study suggested that KT with SE protocol and SKT with SE protocol has a significant effect in improving pressure pain threshold whereas there was non-significant difference found among group on VAS \& NDI in subjects with levator scapulae trigger point in CMNP.

\section{Conflict of interest:}

None

\section{Funding:}

None

Acknowledgments: The authors thanks Director, NILD (Divyangjan) Kolkata for his invaluable help in carrying out the project at the institute campus. We also acknowledge the patients who patiently participated in this study. 
Table 1:-Demographic data in all three groups

\begin{tabular}{|c|c|c|c|c|c|}
\hline & $\begin{array}{c}\text { Group- A } \\
(\text { Mean } \pm \text { SD) }\end{array}$ & $\begin{array}{c}\text { Group- B } \\
(\text { Mean } \pm \text { SD) }\end{array}$ & $\begin{array}{c}\text { Group- C } \\
(\text { Mean } \pm \text { SD) }\end{array}$ & F-value & p- value \\
\hline Female/ Male & $11 / 04$ & $06 / 09$ & $09 / 06$ & & \\
\hline Age & $29.47 \pm 5.85$ & $31.53 \pm 7.80$ & $32.00 \pm 8.37$ & 0.496 & 0.613 \\
\hline VAS & $6.38 \pm 0.77$ & $6.69 \pm 1.16$ & $5.80 \pm 1.14$ & 2.837 & 0.70 \\
\hline PPT & $3.20 \pm 0.23$ & $3.15 \pm 0.16$ & $3.13 \pm 0.29$ & 0.322 & 0.727 \\
\hline NDI & $35.44 \pm 15.09$ & $30.55 \pm 10.48$ & $34.69 \pm 13.52$ & 0.599 & 0.554 \\
\hline
\end{tabular}

Table 2:-Intragroup comparisons after treatment in all the groups. [*= significant difference]

\begin{tabular}{|c|c|c|c|c|}
\hline & & $\begin{array}{c}\text { Pre } \\
(\text { Mean } \pm \text { SD })\end{array}$ & $\begin{array}{r}\text { Post } \\
(\text { Mean } \pm \text { SD })\end{array}$ & P value \\
\hline \multirow{3}{*}{ Group -A } & VAS & $6.38 \pm 0.77$ & $2.58 \pm 1.58$ & $0.00 *$ \\
\hline & PPT & $3.20 \pm 0.23$ & $5.64 \pm 1.29$ & $0.00 *$ \\
\hline & NDI & $35.44 \pm 15.09$ & $13.69 \pm 11.50$ & $0.00 *$ \\
\hline \multirow{3}{*}{ Group -B } & VAS & $6.69 \pm 1.16$ & $2.62 \pm 1.37$ & $0.00 *$ \\
\hline & PPT & $3.15 \pm 0.16$ & $4.93 \pm 0.70$ & $0.00 *$ \\
\hline & NDI & $30.55 \pm 10.48$ & $9.20 \pm 7.99$ & $0.00 *$ \\
\hline \multirow{3}{*}{ Group -C } & VAS & $5.80 \pm 1.14$ & $3.01 \pm 1.44$ & $0.00 *$ \\
\hline & PPT & $3.13 \pm 0.29$ & $4.03 \pm 0.58$ & $0.00 *$ \\
\hline & NDI & $34.69 \pm 13.52$ & $14.97 \pm 12.82$ & $0.00 *$ \\
\hline
\end{tabular}

Table 3:-Intergroup comparisons after treatment in all the groups. [*= significant difference]

\begin{tabular}{|c|c|c|c|c|}
\hline & GROUP 1 (I) & GROUP 2 (J) & MEAN DIFFERENCE (I-J) & P value \\
\hline \multirow{3}{*}{ VAS } & GROUP A & GROUP B & -0.040 & 1.000 \\
\cline { 2 - 5 } & GROUP A & GROUP C & -0.433 & 1.000 \\
\cline { 2 - 5 } & GROUP B & GROUP C & -0.393 & 1.000 \\
\hline \multirow{3}{*}{ PPT } & GROUP A & GROUP B & 0.713 & 0.117 \\
\cline { 2 - 5 } & GROUP A & GROUP C & $1.620^{*}$ & $0.000^{*}$ \\
\cline { 2 - 5 } & GROUP B & GROUP C & $0.907^{*}$ & 0.806 \\
\hline \multirow{3}{*}{ NDI } & GROUP A & GROUP B & 4.487 & 1.000 \\
\cline { 2 - 5 } & GROUP A & GROUP C & -1.283 & 0.471 \\
\cline { 2 - 5 } & GROUP B & GROUP C & -5.770 & \\
\hline
\end{tabular}




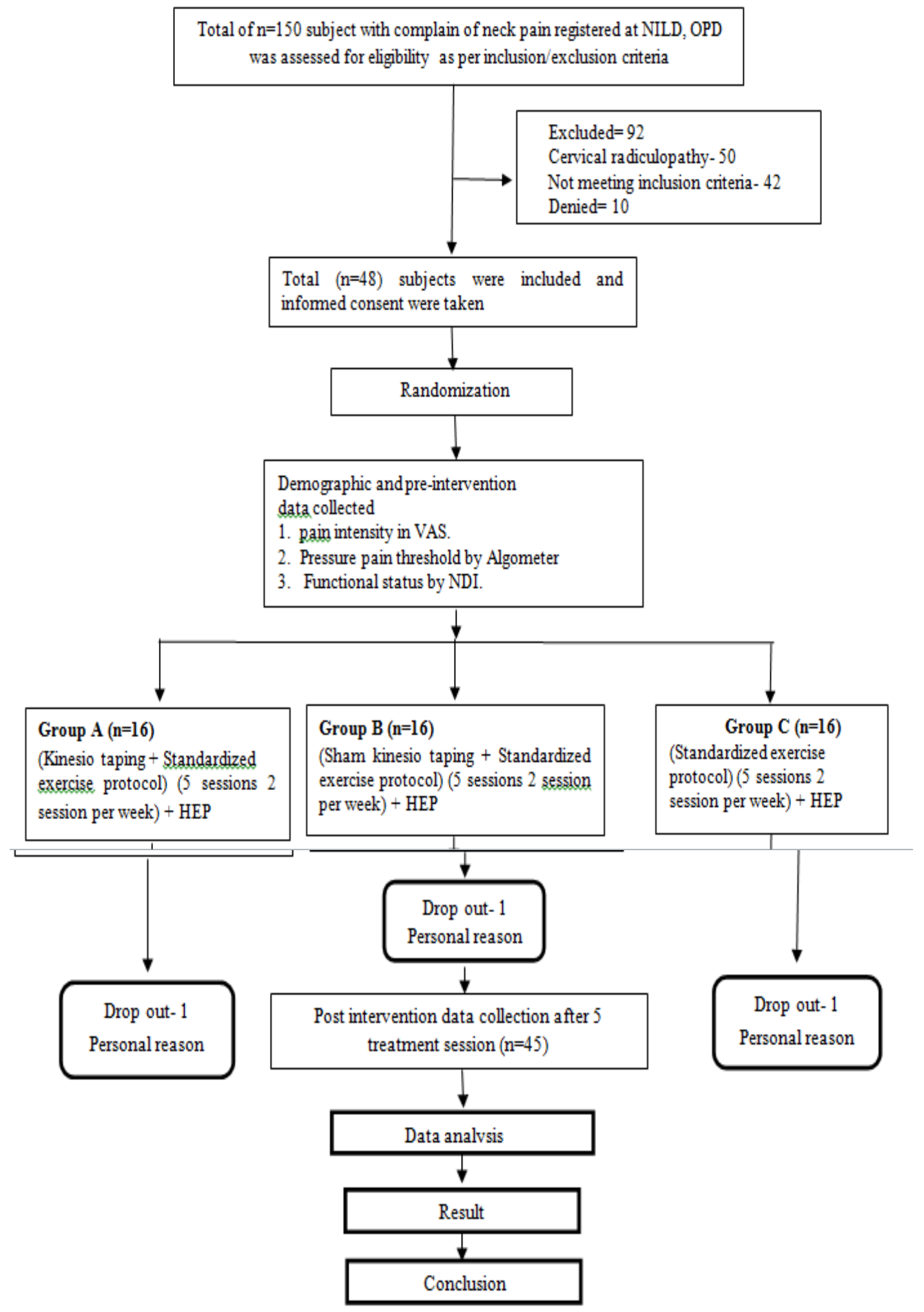

Figure 5.2:-Consort Flow Diagram 


\section{References:-}

1. Misailidou V, Malliou P, Beneka A, Karagiannidis A, Godolias G, Assessment of patients with neck pain: a review of definitions, selection criteria, and measurement tools; Journal of Chiropractic Medicine Jun 2010; 9(2): 49-59.

2. Fejer r, Kyvik Ko, hartvigsen J. the prevalence of neck pain in the world population: a systematic critical review of the literature. Eur spine J 2006; 15:834-48.

3. Hoy D, March L, Woolf A, Blyth F, Brooks P, Smith E, Vos T, Barendregt J, Blore J, Murray C, Burstein R. The global burden of neck pain: estimates from the global burden of disease 2010 study. Annals of the rheumatic diseases. 2014 Jul 1;73(7):1309-15.

4. Chahal RK, Kumar P. Alteration in Activation Pattern of Neck Muscles in Patient with Chronic Neck Pain. International Journal of Head and Neck Surgery 2015; 6(1):1-7.

5. Sjogaard G, Lundberg U, Kadefors R. The role of muscle activity and mental load in the development of pain and degenerative processes at the muscle cell level during computer work. European journal of applied physiology. 2000 Oct 7;83(2):99-105.

6. Chien A, Sterling M. Sensory hypoaesthesia is a feature of chronic whiplash but not chronic idiopathic neck pain. Manual therapy. 2010 Feb 1;15(1):48-53.

7. Lluch E, Nijis J, De Kooning M, Van Dyckl D,Vanderstraeten R, Struyf F, Roussel NA, Prevalence, Incidence, localisation of pathophysiology of myofascial trigger point in the patient with spinal pain, Journal of Manipulative and Physiological Therapeutics.2015 oct; 38(8);587-600.

8. Halski T, Ptaszkowski K, Słupska L, Paprocka-Borowicz M, Dymarek R, Taradaj J, Bidzinska G, Marczynski D, Cynarska A, Rosinczuk J. Short-term effects of kinesio taping and cross taping application in the treatment of latent upper trapezius trigger points: a prospective, single-blind, randomized, sham-controlled trial. Evidence-based complementary and alternative medicine. 2015;2015.

9. Simons DG, Travell JG, Simons LS. Myofascial pain and dysfunction: the trigger point manual, Vol 1, upper half of body. London, Lippincott Williams and Wilkins. 1999; 491-494.

10. Tough EA, White AR, Richards S, Campbell J. Variability of criteria used to diagnose myofascial trigger point pain syndrome- evidence from a review of the literature. The Clinical journal of pain. 2007 Mar 1;23(3):278-86.

11. Alotaibi M, Ayoub A, King T, Uddin S. The effect of kinesio taping in reducing myofascial pain syndrome on the upper trapezius muscle: a systematic review and meta-analysis. European Scientific Journal, ESJ. 2018 Feb $28 ; 14(6)$.

12. El-Abd AM, Ibrahim AR, El-Hafez HM. Efficacy of kinesio taping versus postural correction exercises on pain intensity and axioscapular muscles activation in mechanical neck dysfunction: a randomized blinded clinical trial. The Journal of sports medicine and physical fitness. 2017 Oct;57(10):1311-7.

13. Ay S, Konak HE, Evcik D, Kibar S. The effectiveness of Kinesio Taping on pain and disability in cervical myofascial pain syndrome. Revista brasileira de reumatologia. 2017 Apr;57(2):93-9.

14. Shakeri H, Soleimanifar M, Arab AM, Behbahani SH. The effects of KinesioTape on the treatment of lateral epicondylitis. Journal of Hand Therapy. 2018 Jan 1;31(1):35-41.

15. Ozturk G, Kulcu DG, Mesci N, Şilte AD, Aydog E. Efficacy of kinesio tape application on pain and muscle strength in patients with myofascial pain syndrome: a placebo-controlled trial. Journal of physical therapy science. 2016;28(4):1074-9.

16. El-Abd AM, Ibrahim AR, El-Hafez HM. Efficacy of kinesio taping versus postural correction exercises on pain intensity and axioscapular muscles activation in mechanical neck dysfunction: a randomized blinded clinical trial. The Journal of sports medicine and physical fitness. 2017 Oct;57(10):1311-7.

17. Thomas T.W. Chiu, Tai-Hing Lam, Anthony J. Hedley, A Randomized Controlled Trial on the Efficacy of Exercise for Patients with Chronic Neck Pain. Spine 2004; 30:1:1-7.

18. Parreira PCS, Costa LCM, Takahashi R, Hespanhol Junior LC, da Luz Junior MA, da Silva TM, et al. Kinesio Taping to generate skin convolutions is not better than sham taping for people with chronic non-specific low back pain: A randomised trial. Journal of Physiotherapy 2014; 60:90-6. 